\title{
Magnetic microstructure of sputtered Co-Cr films
}

\author{
P. ten Berge ${ }^{a}$, J.C. Lodder ${ }^{a}$, R. Plöß1 ${ }^{b}$ and J.N. Chapman ${ }^{b}$ \\ ${ }^{a}$ MESA Research Institute, University of Twente, P.O. Box 217, 7500 AE Enschede, The Netherlands \\ ${ }^{b}$ Department of Physics and Astronomy, University of Glasgow, Glasgow G12 8QQ, UK
}

\begin{abstract}
The magnetic microstructure of a thickness series of $\mathrm{Co}_{81} \mathrm{Cr}_{19}$ layers on $\mathrm{Si}_{3} \mathrm{~N}_{4}$ membranes is investigated by modified differential phase contrast (MDPC) microscopy. The development from cross-tie wall structures for a thickness $<25 \mathrm{~nm}$ to more complicated structures for a thickness $>50 \mathrm{~nm}$ is related to the macroscopic VSM measurements and the crystallite orientation determined from electron diffraction experiments.
\end{abstract}

\section{Introduction}

The thickness of magnetic layers for high-density magnetic perpendicular recording applications is decreasing: thinner layers exhibit smoother surfaces, which, for ultralow head-medium spacings, is a requirement to achieve linear recording densities above 5 $\mathrm{kb} / \mathrm{mm}$. Therefore, we studied the development of the micromagnetic structure of $\mathrm{Co}_{81} \mathrm{Cr}_{19}$ layers in the thickness range 5-100 $\mathrm{nm}$.

It has become clear that $\mathrm{Co}-\mathrm{Cr}$ layers are structurally and therefore also magnetically inhomogeneous in their thickness direction [1]; with increasing thickness a magnetically isotropic, in-plane layer develops into a hexagonal, columnar structure with perpendicular magnetic anisotropy.

In order to study the micromagnetic behaviour of $\mathrm{Co}-\mathrm{Cr}$ layers with such small thicknesses, we need to consider the relation between the direction and magnitude of the magnetic anisotropy and the domain wall types connected with them. It is known that thin layers (of the order of the wall width) exhibit Néel walls, while thicker layers typically have Bloch walls. More complicated wall types, such as the cross-tie wall, are found, for instance, in soft magnetic materials such as permalloy (see fig. 1). The dotted and crossed circles denote the so-called Bloch lines, with a magnetization direction out of and into the paper, respectively.

\section{Experimental}

The $\mathrm{Co}_{81} \mathrm{Cr}_{19}$ layers investigated in this paper have been rf-sputter-deposited on $40 \mathrm{~nm}$ thick $\mathrm{Si}_{3} \mathrm{~N}_{4}$ membranes [2]. The hysteresis curves are measured by a VSM. The magnetic induction images have been

Correspondence to: Dr. J.C. Lodder, MESA Research Institute; University of Twente, P.O. Box 217, 7500 AE Enschede, The Netherlands. recorded on a VG HB5 STEM in the Lorentz mode; using an eight-segment detector, we have obtained the induction images by subtracting signals from opposing segments of the outer annular quadrant detector; the difference signals between two such segments is proportional to one component of the Lorentz force, and thus provides quantitative information about the magnetic induction in one direction [3]. The white areas in the induction images denote a magnetic induction to the right and upwards for the $x$ - and $y$-directions, respectively; the opposite is true for the black areas, and any grey level between white and black consequently denotes an intermediate induction direction. The magnetic induction vector mappings have been obtained using a digital image-processing package [4], that converts the linear information about the induction in the $x$ - and $y$-directions into a map containing the local magnetic induction vectors; the size and direction of an arrow corresponds to the magnitude and direction of magnetic induction vector.

\section{Results}

Table 1 gives some relevant magnetic properties of the thickness series $\mathrm{Co}-\mathrm{Cr}$ on $\mathrm{Si}_{3} \mathrm{~N}_{4}$ layers as determined from VSM hysteresis and torque curves. As can be seen from the values of the in-plane $\left(H_{\mathrm{c} \|}\right)$ and perpendicular $\left(H_{\mathrm{c} \perp}\right)$ coercivities, the thinnest layer (5 $\mathrm{nm}$ ) behaves as a soft magnetic layers. The 10 and 25 nm thick layers develop an in-plane coercivity, and from a Co-Cr thickness of $50 \mathrm{~nm}$ quite large coercivities are measured, especially in the perpendicular direction. The $H_{\mathrm{c} \perp} / H_{\mathrm{k}}$ values indicate the type of magnetization reversal [5]; small values are typical for domain wall displacement reversal, while high values $(>10 \%)$ are characteristic of reversal through a rotational mode, exhibiting dot-like domains. From the in-plane remanence ratios $\left(M_{\mathrm{r}} / M_{\mathrm{s}}\right)_{\mathrm{IL}}$ it is seen that even the thicker layers still exhibit a significant initial 


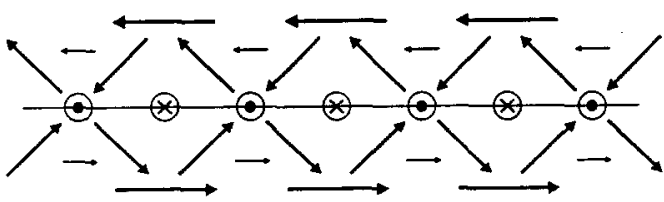

Fig. 1. Schematic of cross-tie wall structure (top view),

Table 1

Magnetic properties of $\mathrm{Co}-\mathrm{Cr} / \mathrm{Si}_{3} \mathrm{~N}_{4}$ series

\begin{tabular}{rllcl}
\hline $\begin{array}{l}h \\
{[\mathrm{~nm}]}\end{array}$ & $\begin{array}{l}H_{\mathrm{c} \|} \\
{[\mathrm{kA} / \mathrm{m}]}\end{array}$ & $\begin{array}{l}H_{\mathrm{c} \perp} \\
{[\mathrm{kA} / \mathrm{m}]}\end{array}$ & $\begin{array}{l}H_{\mathrm{c} \perp} / H_{\mathrm{k}} \\
{[\%]}\end{array}$ & $\left(M_{\mathrm{r}} / M_{\mathrm{s}}\right)_{\mathrm{IL}}$ \\
\hline 5 & $\approx 0$ & $\approx 0$ & 0 & 0.80 \\
10 & 5 & $\approx 0$ & 0 & 0.83 \\
25 & 13 & $\approx 0$ & 0 & 0.85 \\
50 & 28 & 65 & 11 & 0.63 \\
75 & 30 & 88 & 17 & 0.53 \\
100 & 25 & 65 & 14 & 0.53 \\
\hline
\end{tabular}

layer [6], due to the $\mathrm{Si}_{3} \mathrm{~N}_{4}$ substrate, as discussed in ref. [2]. This in-plane initial layer (IL) is also clear from fig. 2, which represents the in-plane hystereses of the 10 and $75 \mathrm{~nm}$ thick layers.

Electron diffraction experiments [2] showed that in the thinnest layers (thickness $<25 \mathrm{~nm}$ ) a certain amount of hcp phase with in-plane orientation of the $c$-axis is present, as indicated by the (101) and (002) diffraction rings. At thicknesses above $50 \mathrm{~nm}$ the crystallites tend to become oriented perpendicular to the film plane, as seen from the (100) direction ring, leaving a relatively minor fraction in the in-plane directions, as also reported in ref. [7]. From bright-field TEM images it is known that the crystal size varies from about $10 \mathrm{~nm}$ diameter for the $10 \mathrm{~nm}$ thick layer to about $30-50 \mathrm{~nm}$ diameter for the $75 \mathrm{~nm}$ thick layer [2]. Finally, the

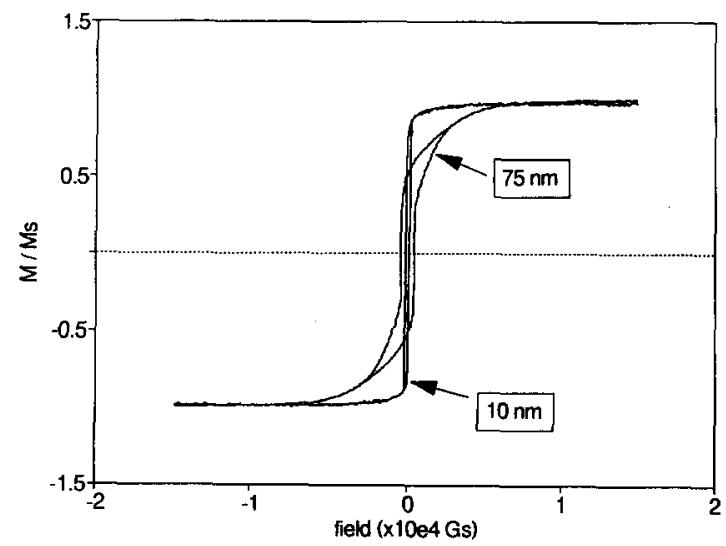

Fig. 2. In-plane hysteresis curves of 10 and $75 \mathrm{~nm} \mathrm{Co-Cr}$ layers.

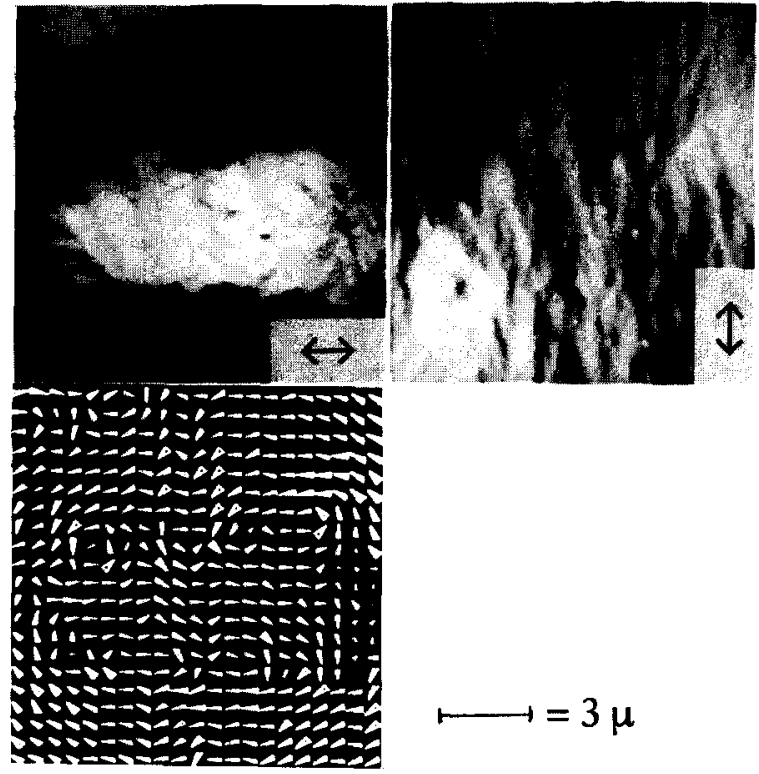

Fig. 3. Overall structure of $10 \mathrm{~nm} \mathrm{Co-Cr}$ layer.

dispersion of the (002) crystal planes, $\Delta \theta_{50}$, was too large to be detectable for the thinner layers, and is about $15^{\circ}$ for the layers thicker than $50 \mathrm{~nm}$.

Thus, these layers do develop a crystal anisotropy in the perpendicular direction, albeit slowly, but their average magnetization anisotropy direction remains in-plane through the demagnetizing forces (the $Q$-factor, $\left(K_{1} / \frac{1}{2} \mu_{0} M_{\mathrm{s}}^{2}\right)$ is about 0.5 for the layers thicker than $25 \mathrm{~nm}$ ).

In the remainder of this paper we will discuss the micromagnetic structure of the 10 and $75 \mathrm{~nm} \mathrm{Co-Cr}$ films mentioned in table 1. Prior to the observations the specimens have been ac-demagnetized by rotating the sample in a decreasing ac field.

Figure 3 provides an impression of the micromagnetic structure at a large scale. In the $x$-direction large domains are observed, while the $y$-direction is characterized by a ripple structure. Such a ripple structure in a soft-magnetic layer is often attributed to (in-plane) random anisotropy variations; this is consistent with our electron diffraction experiments [2]. The difference in induction patterns in $x$ - and $y$-directions may well originate from the way of ac demagnetization of the specimens, where the axis of rotation in the decreasing dc field was about parallel to the $x$-direction, so that the effective ac field in the $x$-direction was very small; the demagnetization in this direction was therefore not complete.

Figure 4 shows a cross-tie wall structure. The crosstie, as well as the surrounding flux-closed vortex structures (see also fig. 1 for a schematic representation of 


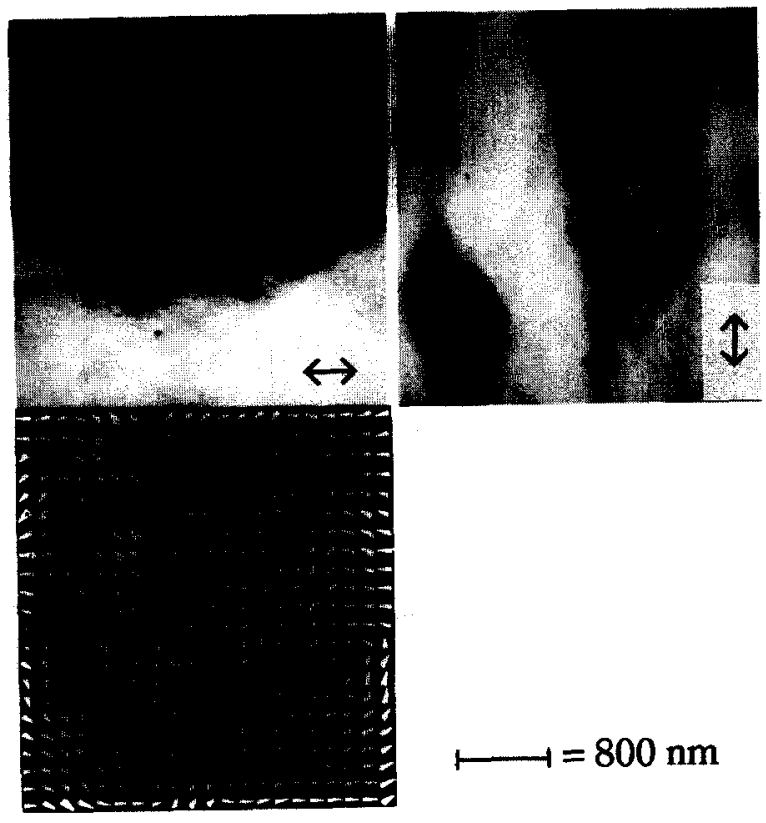

Fig. 4. Typical cross-tie structure in $10 \mathrm{~nm}$ specimen (see also fig. 1).

this structure) can be easily recognized. A higher magnification of the central area of the image in fig. 4 is given in fig. 5. These images and this vector mapping give a clearer view at the actual cross-tie, with its 'singular point of magnetization' (Block line) at the

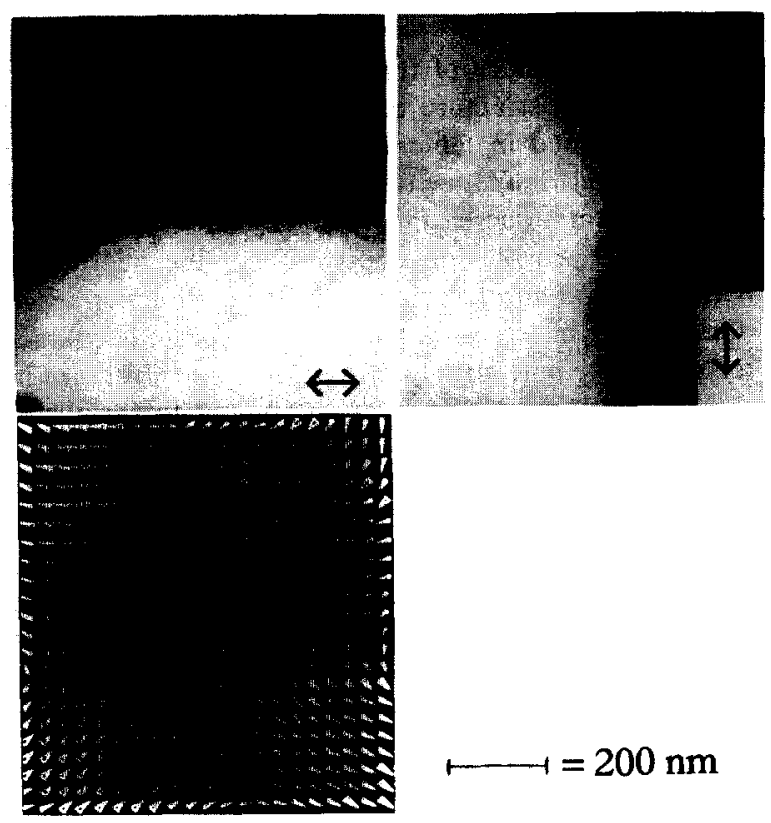

Fig. 5. Detailed image of cross-tie wall in fig. 4.

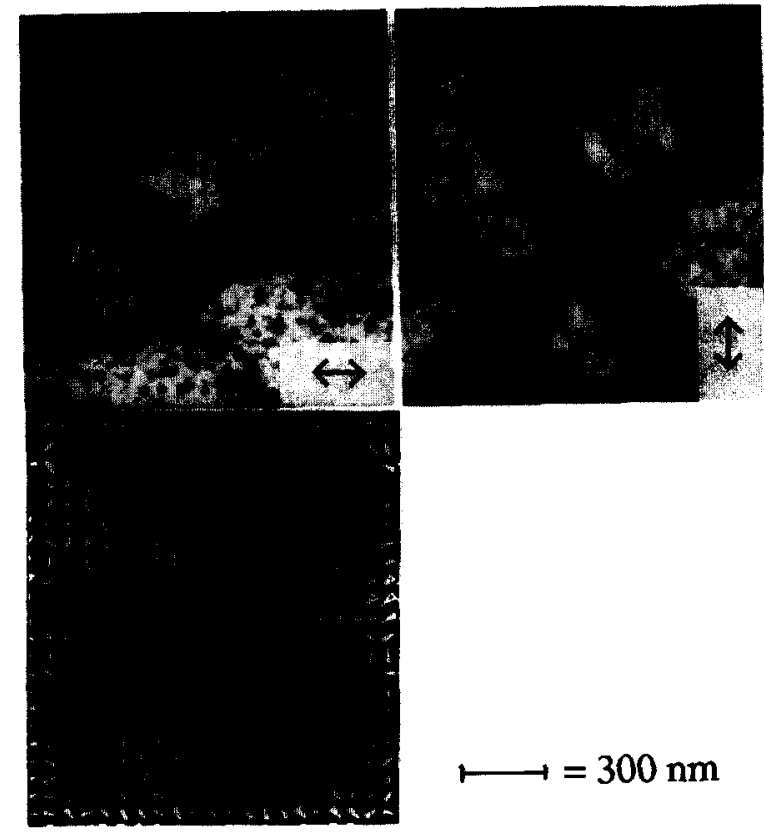

Fig. 6. Detailed image of stripes in $75 \mathrm{~nm} \mathrm{Co-Cr}$ layer.

center; it is assumed that the magnetic induction direction at this point is into or out of the paper.

Although the way of demagnetization was similar for the $75 \mathrm{~nm}$ thick specimen, no difference in $x$ - and $y$-induction was observed in the overall structure at low magnification. This is attributed to the fact that locally the easy direction of magnetization has come out of the plane of the specimen through the crystalline anisotropy, as interpreted from the increased intensity of the crystal planes (relative to thinner layers) in the [100] direction in the electron diffraction experiments [2]. Now the effective ac field acting in the direction of magnetization is larger, leaving a better demagnetized specimen. The vector mapping of these induction images has decreased in information value, since the period of induction changes is too small to convert to vectors. Therefore in fig. 6 a higher magnification of this area is shown.

The induction images show a pattern with randomly-oriented stripes; it is noted that the images in fig. 6 result from the deflection of the electrons through the whole layer (as all magnetic induction images). Therefore the images may well consist of a superposition of different types of surface and bulk domains and domain walls. Besides this, the induction images and the vector mapping are less conclusive than those for the $10 \mathrm{~nm}$ layer, because of the increased disturbance by crystallite contrast (significantly larger crystal size) and possibly because of the (locally) out-of-plane mag- 
netic induction. Furthermore, the dimension of the magnetic contrast is smaller; the number of pixels that is integrated over to calculate a magnetic induction vector may contain different grey levels, i.e. different induction directions.

\section{Conclusions}

It has been shown that $\mathrm{Co}-\mathrm{Cr}$ layers on $\mathrm{Si}_{3} \mathrm{~N}_{4}$ thinner than $10 \mathrm{~nm}$ behave soft-magnetically, as derived from the macroscopic VSM hysteresis curves. High-resolution micromagnetic observations also demonstrate this through the cross-tie wall and the flux-closed vortex structures, that are familiar from soft-magnetic thin permalloy layers. Such layers are known to establish a magnetization reversal through domain wall displacement, which is supported by the very small $H_{\mathrm{c} \perp} / H_{\mathrm{k}}$ values. Cross-tie wall structure observations have been reported earlier (observed at low magnification by TEM in the Fresnel mode) in thin $\mathrm{Co}-\mathrm{Cr}$ layers, prepared by rf-sputtering on glass or collodion [8] as well as dc-magnetron sputtering on carbon substrates [9]. An increase in thickness of the layer (thickness $>50 \mathrm{~nm}$ ) leads to a more complicated domain (wall) structure, with smaller dimensions and magnetic induction patterns that are shaped as stripes at remanence after an ac-demagnetization cycle. No dot-like domains have been observed, as could be expected from the high $H_{\mathrm{c} \perp} / H_{\mathrm{k}}$ values, or as reported in refs. [8,9]. Considering this together with the high perpendicular coercive field, the out-of-plane crystalline anisotropy and larger crystallite size, it is likely that the magnetization reversal proceeds via a mechanism involving a strongly pinned domain wall displacement.

The authors thank the CAMST (Community Action on Magnetic Storage Technology) EC stimulation project for financial support. Furthermore P.t.B. and J.C.L. acknowledge the Foundation for Fundamental Research on Matter and R.P. acknowledges the EC support, contract no. BREU 900320.

\section{References}

[1] C.P.G. Schrauwen and J.P.C. Bernards, J. Magn. Soc. Jpn. 13 (S1) (1989) 669.

[2] P. ten Berge, S. Porthun, J.C. Lodder and Th.J.A. Popma, J. Magn. Magn. Mater. 113 (1992).

[3] J.N. Chapman, S. McVitie and S.J. Hefferman, J. Appl. Phys. 69 (1991) 6078.

[4] J. Zweck, J.N. Chapman, S. McVitie and H. Hoffmann, J. Magn. Magn. Mater. 104-107 (1992) 315.

[5] C.-Z. Li and J.C. Lodder, IEEE Trans. Magn. MAG-23 (1987) 2260.

[6] J.C. Lodder and C.-Z. Li, IEEE Trans. Magn. MAG-24 (1988) 1889.

[7] J.C. Lodder and T. Wielinga, IEEE Trans. Magn. MAG-20 (1984) 57.

[8] M. Ohkoshi, H. Toba, S. Honda and T. Kusuda, J. Magn. Magn. Mater. 35 (1983) 266.

[9] J.W. Lee, B.G. Demczyk, K.R. Mountfield and D.E. Laughlin, J. Appl. Phys. 63 (1988) 2905. 